\title{
Normalized Contact Force to Minimize "Electrode-Lead" Resistance in a Nanodevice
}

\author{
Seung-Hoon Lee, Jun Bae, ${ }^{\dagger}$ Seung Woo Lee, ${ }^{\dagger}$ and Jae-Won Jang* \\ Department of Physics, Pukyong National University, Busan 608-737, Korea. *E-mail: jjang@pknu.ac.kr \\ ${ }^{\dagger}$ School of Chemical Engineering, Yeungnam University, Gyeongsan 712-749, Korea \\ Received March 27, 2014, Accepted April 22, 2014
}

\begin{abstract}
In this report, the contact resistance between "electrode" and "lead" is investigated for reasonable measurements of samples' resistance in a polypyrrole (PPy) nanowire device. The sample's resistance, including "electrodelead" contact resistance, shows a decrease as force applied to the interface increases. Moreover, the sample's resistance becomes reasonably similar to, or lower than, values calculated by resistivity of PPy reported in previous studies. The decrease of electrode-lead contact resistance by increasing the applying force was analyzed by using Holm theory: the general equation of relation between contact resistance $\left(R_{H}\right)$ of two-metal thin films and contact force $\left(R_{H} \propto 1 / \sqrt{F}\right)$. The present investigation can guide a reliable way to minimize electrode-lead contact resistance for reasonable characterization of nanomaterials in a microelectrode device; $80 \%$ of the maximum applying force to the junction without deformation of the apparatus shows reasonable values without experimental error.
\end{abstract}

Key Words : Contact resistance, Contact force, Holm theory, Ag paste, Electrode-lead contact

\section{Introduction}

In order to have efficient performance of nanodevices, the junction between microelectrode and sample nanomaterials (as well as properties of nanomaterials) is important. A good junction, such as "Ohmic contact", is favorable for high quality nanomaterials-based applications. In real applications, nanodevices should be connected by electrical leads into apparatus that monitors performance of the nanodevice. Besides the nanomaterials-electrode junction, good an "electrode-lead" junction is necessary for optimal performance of nanodevices. Efforts to achieve reliable junctions in nanodevices can be considered as obtaining lower metal-metal or metal-semiconductor contact resistance. It has been reported that the contributions to contact resistance include microscale geometry, ${ }^{1-5}$ oxide layer of semiconductor materials, ${ }^{6,7}$ difference of Fermi energy between nanomaterials and electrodes, ${ }^{8}$ and contact force between two metal films. ${ }^{3}$ Furthermore, dependence on contact force ${ }^{3,9}$ was investigated, as well as dependence on contact length, ${ }^{10}$ to understand characteristics of contact resistance. It seems that contact resistance can be altered by many elements, and not all of these are easy to be controlled. Challenges to minimize contact resistance have been investigated by several methods, including ultra-low contact resistance by epitaxial interfacing of nanowires to electrodes ${ }^{11}$ and pre-process to remove oxide layer before deposition of nanomaterials on electrodes. ${ }^{6,7}$

Conventional photolithography and/or e-beam lithography (EBL) are generally used to make microelectrodes in nanodevices. ${ }^{12-14}$ Microelectrodes are integrated into apparatus to supply signals and monitor response of nanomaterials. Usually, conductive pastes are used to connect between microelectrodes and electrical leads of apparatus. ${ }^{15,16}$ However, lower electrical conductivity, poor impact strength, and decreasing conductivity by humidity aging or normal use condition have been reported in reliability testing using silver paste as compared with tin-lead solder. ${ }^{17}$ As it is difficult to use tin-lead soldering in microelectrodes for lead connections, more efforts to develop electrode-lead junctions better than silver pasting are needed. In addition, although the electrode-lead contact resistance caused by connection of microelectrodes to apparatus is also an empirically important factor, there have been few reports on "electrode-lead" contact resistance.

In the present study, resistance of nanodevices, composed of polypyrrole (PPy) nanowires (NWs) loaded on Au microelectrodes, was measured with non-silver paste electrodelead junction in room conditions. Ag wires were physically contacted on the $\mathrm{Au}$ microelectrodes to transfer electrical signals between PPy NWs and an electrometer, and force was applied to the normal direction of the wire-electrode contact. The force dependent resistance of the device showed that electrode-lead contact resistance can be effectively minimized by increase of the applying force in accordance with Holm theory; this demonstration can be utilized for reasonable characterization of nanomaterials in microelectrode devices.

\section{Experimental}

The experimental set-up for the force dependent electrodelead contact resistance is displayed in Figure 1. PPy NWs were fabricated by electrochemical deposition into aluminum oxide (AAO) template using $\mathrm{ClO}_{4}$ as a count ion. The PPy NWs were dispersed on a piece of $\mathrm{Au}$ microelectrodes patterned $\mathrm{SiO}_{\mathrm{x}} / \mathrm{Si}$ substrate $(12.5 \mathrm{~mm} \times 12.5 \mathrm{~mm} \times 0.55 \mathrm{~mm})$, 

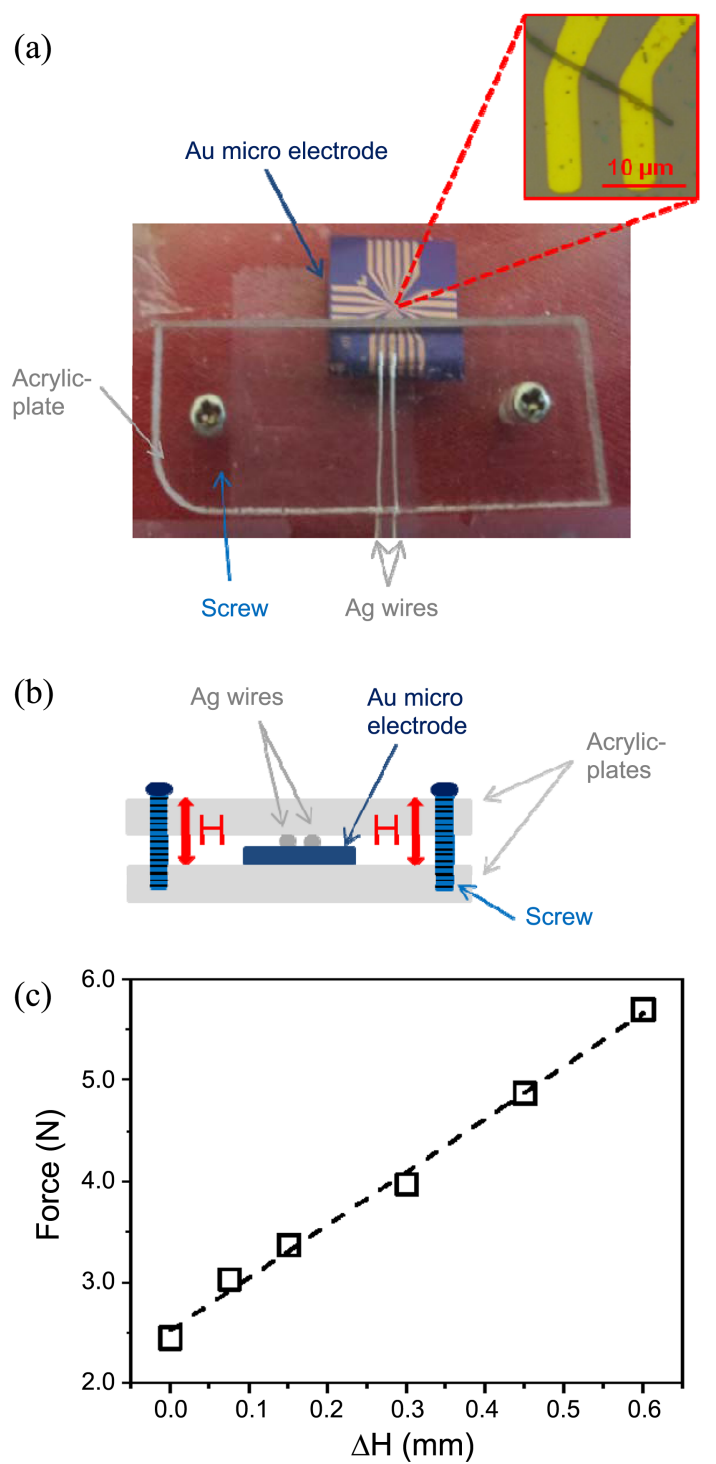

Figure 1. (a) A snap-shot image and (b) scheme of experimental set-up for contact force dependent resistance measurement of a PPy NW device. The inset in (a) is a zoomed-in optical microscope image of PPy NW loaded area. (c) Applying force curve dependent on displacement $(\Delta \mathrm{H})$ of the distance between the acrylic plates $(\mathrm{H})$.

as shown in the inset image in Figure 1. For the electrodelead junction, pure Ag wires (Dia.: $0.25 \mathrm{~mm}, 99.99 \%$, GoodFellow, Inc.) were put on the $\mathrm{Au}$ microelectrodes without any conductive paste or adhesive. Instead, the Ag wires physically contact onto the microelectrodes sandwiched between screws connected to two acrylic plates. Forces applying to the "Au electrode-Ag lead" junction can be adjusted by rotations of the screws, which are equally rotated (Figure 1(b)). Measurement of the applying force was carried out by a force sensor (Cl-6537, Pasco scientific, Inc.; see Supporting Information). As represented in Figure 1(c), the applying force shows a linear dependence on the distance between the acrylic plates $[\mathrm{H}$ and $\Delta \mathrm{H}$ in Figure 1(b): $\Delta \mathrm{H}$ means displacement of $\mathrm{H}]$. The force changes linearly from 2.45 to $5.7 \mathrm{~N}$ as the $\Delta \mathrm{H}$ value increases from
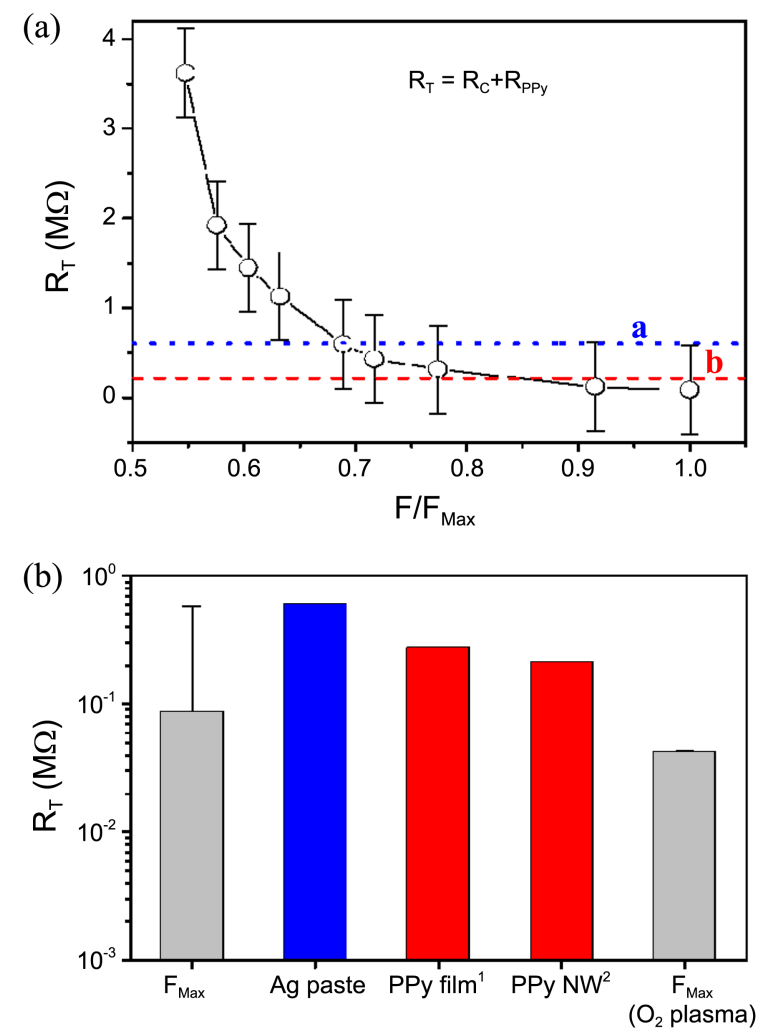

Figure 2. (a) Electrode-lead contact resistance vs. contact force: total resistance of the device $\left(R_{T}\right)$ dependent on contact force normalized by the maximum contact force $\left(F / F_{M a x}\right)$. "a" is resistance of a PPy NW device with Au electrode-Ag lead junction using silver paste, and "b" is resistance of the PPy NW device calculated by using resistivity of PPy NW (undoped). $R_{T}$ is represented by the sum of contact resistance $\left(R_{C}\right)$ in the device and resistance of PPy NW $\left(R_{P P y}\right)$. (b) The bar graphs of $R_{T}$ with the maximum contact force $\left(F_{\text {Max }}\right)$, junctioned by $\mathrm{Ag}$ paste, and the maximum contact force sequentially after $\mathrm{O}_{2}$ plasma treatment $\left[F_{\text {Max }}\left(\mathrm{O}_{2}\right.\right.$ plasma $\left.)\right]$; and the bar graphs of resistances of a PPy NW device using resistivity of PPy film (PPy film ${ }^{1}$ ) and PPy NW (PPy $\left.\mathrm{NW}^{2}\right)$. ${ }^{1}$ Resistivity of PPy film $\left(\mathrm{ClO}_{4} \text { as a count ion }\right)^{20}$; ${ }^{2}$ Resistivity of PPy NW (undoped). ${ }^{13}$

0.0 to $0.6 \mathrm{~mm}$. From this linear dependence, applying forces during experiments were gauged and extrapolated by the $\mathrm{H}$ values with the screws rotating. Resistance of PPy NW devices were measured with a source-meter (2400, Keithley Instrument, Inc.). For a control experiment, resistance of PPy NW device with a silver paste (Dotite D-500) contact "Au electrode-Ag lead" junction was also measured. $\mathrm{O}_{2}$ plasma treatments of $\mathrm{Au}$ microelectrodes were carried out by a plasma cleaner (Harrick Plasma), with $11 \mathrm{~W}$ for $10 \mathrm{~min}$. All PPy NW devices were prepared with the same configuration (only one strand loading, equal length of PPy NW bridging between the microelectrodes) to minimize resistance deviation caused by PPy NW in different devices.

\section{Results and Discussion}

According to configuration of the PPy NW device shown in Figure 1(a), total resistance of the device $\left(R_{T}\right)$ will be 
straightforwardly represented by Eq. (1):

$$
R_{T}=R_{C}+R_{P P y}
$$

where $R_{P P y}$ is resistance of PPy $\mathrm{NW}$ and $R_{C}$ is contact resistance originated from nanomaterials-electrode contact and/or electrode-lead contact. In the case of a PPy NW device put on $\mathrm{Au}$ microelectrodes, Ohmic I-V curves were reported. ${ }^{18,19}$ Therefore, $R_{C}$ can be regarded as mainly contributed by metal-metal junction ( $\mathrm{Au}$ electrode-Ag wire lead), not by semiconductor-metal junction (PPy NW-Au microelectrode; Figure S2). The $R_{T}$ was measured with different forces that are applying to the normal direction of "Au electrode-Ag lead" junction (electrode-lead contact resistance $v s$. contact force). Figure 2(a) shows a decrease of $R_{T}$ as the contact force between the Au electrode-Ag lead" junction $(F)$ increases; the $\mathrm{x}$-axis of Figure 2(a) is normalized by the maximum contact force $\left(F_{M a x}\right)$ to the junction without deformation of the acrylic plates. Decrease of $R_{T}$ seems saturated in relatively strong contact force region $(F /$ $\left.F_{\text {Max }} \sim 0.8\right)$. The dotted line of "a" in Figure 2(a) is resistance of a PPy NW device with $\mathrm{Au}$ electrode-Ag lead junction using silver paste. In addition, resistance of the PPy NW device calculated by using resistivity of PPy NW (undoped) ${ }^{13}$ is displayed as the dashed line of " $b$ " in Figure 2(a). In Figure 2(b), $R_{T}$ obtained by different cases are compared using a bar graph, where $R_{T}$ were measured with or without $\mathrm{O}_{2}$ plasma treatment on the microelectrodes before making junction [denoted by $F_{M a x}$ and $F_{M a x}\left(\mathrm{O}_{2}\right.$ plasma), respectively]. "Ag paste" and "PPy NW" in Figure 2(b) represent "a" and "b" of Figure 2(a), respectively, while "PPy film" is resistance of a PPy NW device calculated by using resistivity of PPy film $\left(\mathrm{ClO}_{4}\right.$ as a count ion) reported in the literature. ${ }^{20}$ Figure 2(b) shows that the "Ag paste" is larger than the "PPy film" and "PPy NW," which means the Au electrode-Ag lead junction using silver paste does not effectively reduce the junction resistance. On the other hand, the $F_{M a x}$ and $F_{M a x}\left(\mathrm{O}_{2}\right.$ plasma $)$ are smaller than the "PPy film" and "PPy NW," which indicates that resistivity of our PPy NWs $\left(\mathrm{ClO}_{4}\right.$ as a count ion) is superior to that of PPy film $\left(\mathrm{ClO}_{4}\right.$ as a count ion) and PPy NW (undoped). This shows that the junction resistance is effectively reduced by contact force applying without using silver paste. In addition, the junction resistance can be reduced more by means of $\mathrm{O}_{2}$ plasma treatment of the $\mathrm{Au}$ microelectrodes.

The contact force dependence of $R_{T}$ in Figure 2(a) can be explained by traditional contact theory in two-flat-metal-thin films, so it called "Holm theory." In general, contact resistance in Holm theory, $R_{H}$, can be described by Eq. (2): ${ }^{3}$

$$
R_{H}=\frac{\rho_{1}}{4 a}+\frac{\rho_{2}}{4 a},
$$

where $a$ is the contact spot radius, $\rho_{1}$ and $\rho_{2}$ are electrical resistivity of each metal film (regard the metal films as metal 1 and metal 2, respectively). As the contact spot between two films becomes larger by increasing perpendicularlyapplied contact force between the two films, the contact spot radius with applying force - that is Holm radius $\left(a_{H}\right)$ - can be represented by the contact force $(F)$ and hardness $(H)$ of material with holding the contact force: ${ }^{3}$

$$
a_{H}=\sqrt{\frac{F}{\pi H}} .
$$

From Eqs. (2) and (3), the relation between the contact force and the contact resistance $R_{H}$ can be obtained by Eq. (4):

$$
R_{H} \propto \frac{1}{\sqrt{F}}
$$

The Au electrode-Ag lead junction can be approximated as a junction between two-flat-metal-thin films; contact spot between Ag wire and Au electrode is changed by the contact force between the Au electrode-Ag lead junction $(F)$. The contact resistance, $R_{C}$, of the Au electrode-Ag lead junction can be systematically measured by putting two identical Ag wires on an electrically connected Au electrode (Figure S3). The contact force dependence of $R_{C}$ can be analyzed with normalized contact force $\left(F_{N}\right)$ :
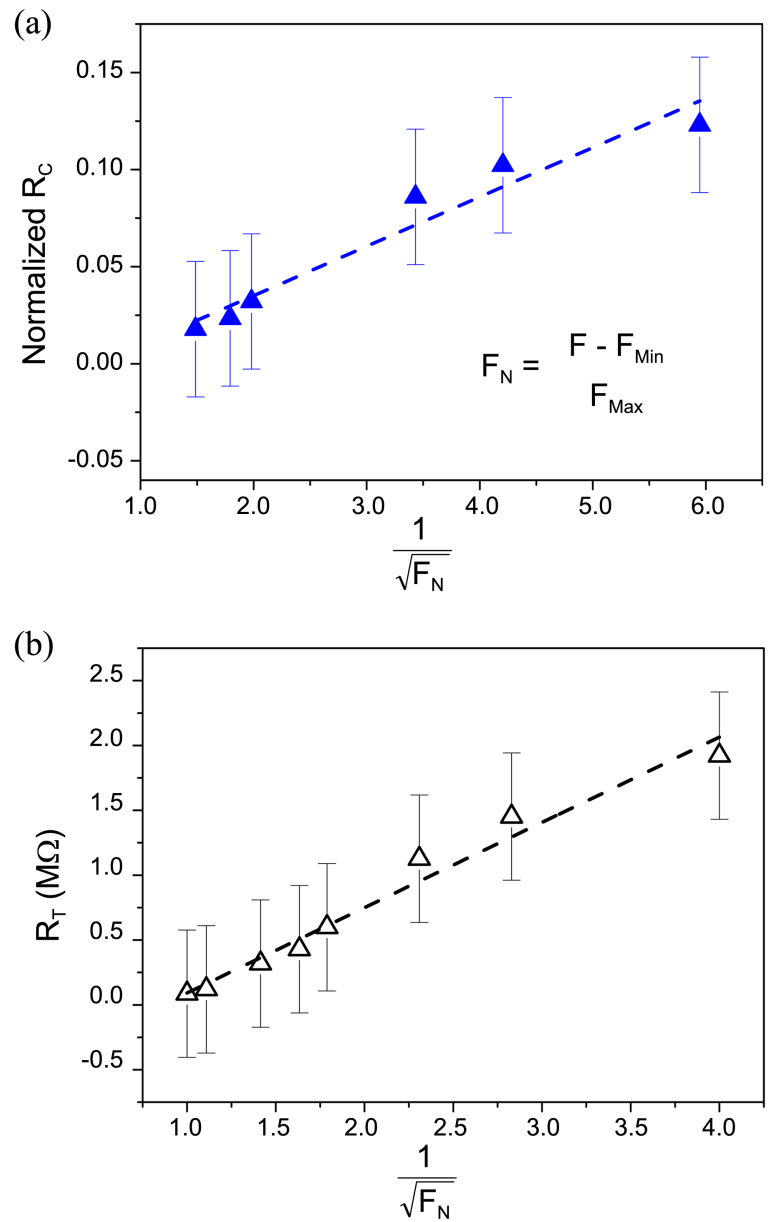

Figure 3. Graphs of (a) normalized $R_{C}$ and (b) $R_{T}$ dependent on $1 / \sqrt{F_{N}}$; both show a linear correlation. The normalized applying force $\left(F_{N}\right)$ is determined by the equation in (a): the $F_{\text {Max }}$ and $F_{\text {Min }}$ are the maximum and minimum contact force during the measurement, respectively. 


$$
R_{H} \propto \frac{1}{\sqrt{\frac{F-F_{M i n}}{F_{\text {Max }}}}}=\frac{1}{\sqrt{F_{N}}},
$$

where $F_{\text {Min }}$ is the minimum contact force during the measurement; resistance cannot be measured by the electrometer if too weak a force is applied to the Au electrode-Ag lead junction. Figure 3(a) represents that normalized $R_{C}$ linearly increases with increase of $1 / \sqrt{F_{N}}$ as expected in Eq. (5). The normalized $R_{C}$ was obtained by dividing by the minimum contact resistance; the minimum contact resistance was determined by the stable contact resistance with the contact force larger than $F_{M a x}$ applied. Due to the relation with $F_{N}$ [shown in Figure 3(a)], the $R_{C}$ can be qualitatively regarded as contact resistance governed by Holm theory.

Moreover, the $R_{T}$ also shows linear dependence on $1 / \sqrt{F_{N}}$ as shown in Figure 3(b). Because the $R_{T}$ is defined as Eq. (1), the contact resistance $R_{C}$ will be more dominant than $R_{P P y}$ (resistance of PPy NW) in the PPy NW device during the experiment. If $R_{C}$ is efficiently reduced, $R_{T}$ will be dominated by $R_{P P y}$. Such an efficient reduction of $R_{C}$ was accomplished by applying contact force to the junction as shown in Figure 2(b); $R_{T}$ shows the even lower resistance than reported values. According to force dependence of $R_{T}$ curve shown in Figure 2(a), $>80 \%$ of the maximum force is necessary to efficiently reduce contact resistance in the PPy $\mathrm{NW}\left(\mathrm{ClO}_{4}\right.$ as count ion) device. We successfully demonstrated that electrical properties of nanodevice can be reasonably measured by efficient contact force without silver paste soldering.

\section{Conclusion}

This letter investigates the total resistance $R_{T}$ of a nanodevice as well as the contact resistance $R_{C}$ of electrode-lead junction behavior like contact resistance ruled by Holm theory. Based upon this observation, it was successfully demonstrated to minimize the experimental error of contact resistance originated from electrode-lead junction. In particular, the measured resistance with at least $80 \%$ of the maximum applying force to the junction without deformation of the apparatus shows reasonable values without experimental error such as riskily high contact resistance. This investigation can suggest a reasonable characterization of nanomaterials in a microelectrode device to minimize electrode-lead contact resistance.

Acknowledgments. J.-W. Jang acknowledges that this work was supported by a Research Grant of Pukyong National University (2014 year).

\section{References}

1. Mikrajuddin, A.; Shi, F. G.; Kim, H. K.; Okuyama, K. Mater. Sci. Semicond. Process 1999, 2, 321-327.

2. Nakamura, M. IEEE Trans. Compon., Hybrids, Manuf. Technol. 1993, 16, 339-43.

3. Read, M. B.; Lang, J. H.; Slocum, A. H.; Martens, R. Electr. Contacts 2009, 55th, 300-306.

4. Sano, Y. Journal of Applied Physics 1985, 58, 2651-2654.

5. Timsit, R. S. Components and Packaging Technologies, IEEE Transactions on 2010, 33, 636-642.

6. Jeon, D. Y.; Kim, K. H.; Park, S. J.; Huh, J. H.; Kim, H. Y.; Yim, C. Y.; Kim, G. T. Appl. Phys. Lett. 2006, 89, 023108/1-023108/3.

7. Sourribes, M. J. L.; Isakov, I.; Panfilova, M.; Warburton, P. A. Nanotechnology 2013, 24, 045703, 6 pp.

8. Kolesnik-Gray, M. M.; Lutz, T.; Collins, G.; Biswas, S.; Holmes, J. D.; Krstic, V. Appl. Phys. Lett. 2013, 103, 153101/1-153101/3.

9. Sawada, S.; Shimizu, K.; Shimada, S.; Hattori, Y. SEI Tekunikaru Rebyu 2010, 177, 36-42.

10. Lan, C.; Srisungsitthisunti, P.; Amama, P. B.; Fisher, T. S.; Xu, X.; Reifenberger, R. G. Nanotechnology 2008, 19, 125703/1-125703/ 7.

11. Chaudhry, A.; Ramamurthi, V.; Fong, E.; Islam, M. S. Nano Lett. 2007, 7, 1536-1541

12. Salfi, J.; Philipose, U.; de, S. C. F.; Aouba, S.; Ruda, H. E. Appl. Phys. Lett. 2006, 89, 261112/1-261112/3.

13. Shen, J.; Chen, Z.; Wang, N.; Yan, H.; Shi, G.; Jin, A.; Gu, C. Appl. Phys. Lett. 2006, 88, 253106/1-253106/3.

14. Stern, E.; Cheng, G.; Young, M. P.; Reed, M. A. Appl. Phys. Lett. 2006, $88,053106 / 1-053106 / 3$.

15. Leong, C.-K.; Chung, D. D. L. J. Electron. Mater. 2004, 33, 203206.

16. Wang, S.; Pang, D. S.; Chung, D. D. L. J. Electron. Mater. 2007, 36, 65-74.

17. Wong, C. P.; Moon, K.-S.; Li, Y. Nano-Bio-Electronic, Photonic and MEMS Packaing; Springer US: 2010; 19.

18. Jang, J.-W.; Sanedrin, R. G.; Senesi, A. J.; Zheng, Z.; Chen, X.; Hwang, S.; Huang, L.; Mirkin, C. A. Small 2009, 5, 1850-1853.

19. Qin, L.; Park, S.; Huang, L.; Mirkin, C. A. Science 2005, 309, 113-115.

20. Singh, R. K.; Kumar, A.; Agarwal, K.; Kumar, M.; Singh, H. K.; Srivastava, P.; Singh, R. J. Polym. Sci., Part B: Polym. Phys. 2012, 50, 347-360. 